\title{
Methoden der Stosswellenerzeugung und -messung
}

\author{
W.Eisenmenger
}

\author{
1.Physikalisches Institut der Universität Stuttgart
}

\begin{abstract}
Grundlagen
Stoßwellen, Schlagwellen oder Knallwellen sind Druckwellen hoher Amplitude, deren Ausbreitungsgeschwindigkeit die Schallgeschwindigkeit aufgrund von Nichtlinearitäten des Mediums übersteigt. Sofern nicht schon bei der Erzeugung der StoBwelle, z.B. durch Detonation, der Druckanstieg als steile Wellenfront vorliegt, entsteht eine steile StoBfront auch während der Ausbreitung einer Uberdruckwelle als Folge der Aufsteilung aufgrund des Anstiegs der Schallgeschwindigkeit mit wachsender Amplitude.
\end{abstract}

Während die Physik und Technik der StoBwellen in Gasen ein umfangreiches und weit erforschtes Gebiet darstellt, hat die StoBwellenausbreitung in Flüssigkeiten in jüngerer Zeit durch die medizinische Anwendung bei der extrakorporalen Steintherapie $/ 1 /, / 2 /$ besonderes Interesse gefunden.

Bei dieser neuen Methode werden StoBwellen oder auch zunächst nur Druckwellen fokussierend in Wasser abgestrahlt, über eine Koppelmembran oder auch, bei direktem Kontakt, in den Körper des Patienten eingeleitet und bei weiterer Ausbreitung im Gewebe auf den zu zerkleinernden Stein fokussiert. Für die Zertrümmerungswirkung werden sowohl die durch den steilen Druckanstieg im Stein erzeugten Druck und Schubspannungen als auch die nach der Reflexion der durchlaufenden Welle entstehenden Zugspannungen (Abplatzeffekt), vergl. $/ 1 /, 12 /$, verantwortlich gemacht. Kürzlich wurde jedoch auch die Wirkung der Kavitation durch Unterdruckanteile der Welle bei der Gallensteinzertrümmerung / $3 /$ nachgewiesen. Ebenso ist zu vermuten, daB Schmerzentstehung und gelegentliche Gewebsschädigung auf unterdruckbedingte Kavitation zurückzuführen sind.

Somit kommt neben der StoBwelle selbst, d.h. dem positiven Teil der Druckwelle auch negativen Druckanteilen im Gefolge des Uberdruckes eine besondere Bedeutung zu. Diese negativen Druckanteile im Feld der fokussierten Welle können für den linearen akustischen Fall grundsätzlich /4/ nicht vermieden werden. Selbst wenn in einem definierten Querschnitt, z.B. an der Generatoroberfläche, ein reiner Uberdruckpuls vorliegt, treten bei der weiteren Ausbreitung im AnschluB an den Uberdruckpuls negative Druckanteile durch Randbeugungswellen auf, wobei das gesamte Druckintegral an jeder Stelle des Wellenfeldes, d.h. auch im Brennpunkt verschwindet. Dort ist das Resultat der Beugungsrechnung beson- ders einfach, da der resultierende Druck im Brennpunkt der zeitlichen Ableitung der ursprünglichen, d.h. erregenden Druckfunktion entspricht, vergl. 14/. Einem steilen Druckanstieg und langsamen Druckabfall im erzeugenden Querschnitt entspricht somit eine kurze, hohe, positive Druckspitze im Fokus mit einem nachfolgenden langen, jedoch schwachen negativen Druckpuls.

Bei einem zeit-symmetrischen Druckverlauf im erzeugenden Querschnitt /4/ ergibt sich eine antisymmetrische Druckfunktion im Fokus, d.h. dem Uberdruckpuls folgt unmittelbar ein entgegengesetzt gleich hoher Unterdruckpuls. Sofern Kavitation für die Steinzertrümmerung wesentlich ist 13/, kann die Stärke des kavitationsauslösenden Unterdruckimpulses somit leicht durch die Pulsform im erzeugenden Generatorquerschnitt eingestellt werden.

Ein weiterer einfacher und interessanter Grenzfall betrifft die Druckzeitfunktion auf der Generatorachse zum Fokus. Hier trifft die Randbeugungswelle jeweils später ein als das direkte Signal. Da die Laufzeit vom Rand zur Achse jedoch einen einheitlichen Wert besitzt, ist das Signal der Randbeugungswelle entgegengesetzt gleich groB zur direkten Welle. Dies folgt aus der bereits genannten Bedingung, daß das gesamte Druck-Zeit-Integral verschwindet.

Auf einen kurzen direkten Uberdruckimpuls folgt daher ein entgegengesetzt gleicher Unterdruckimpuls. Letzterer fuhrt zu Kavitation mit möglichen Nebenwirkungen. Eine Reduzierung der achsennahen Amplitude der Randbeugungswelle gelingt durch eine radial abnehmende Anregungsamplitude (Randabschattung) $/ 4 /$ im erzeugenden Querschnitt, d.h. einer Verteilung der Randbeugungswelle auf ein möglichst großes Laufzeitintervall.

Diese Aussagen gelten zunächst für den Bereich der linearen Akustik. Auch bei Berücksichtigung der endlichen Druckamplituden und der Entstehung von Stoßwellen, bilden die linear-akustischen Lösungen jedoch weiter wichtige Näherungen. Hinsichtlich des meBbaren Unterdrucks treten jedoch durch Kavitation starke Abweichungen gegenüber der akustischen Näherung auf, da durch das kavitationsbedingte AufreiBen der Flüssigkeit der Unterdruckanteil stark geschwächt wird.

Auch hier kann jedoch durch Reduktion der Unter- 
druckwelle, d.h. Randabschattung, die Kavitation mit den entsprechenden Nebenwirkungen reduziert werden.

\section{Erzeugung von Stoßwellen in Flüssigkeiten}

StoBwellen höchster Amplitude entstehen in Flüssigkeiten durch Detonation $/ 5 /$. Bei Verwendung von Zündpillen, vergl. $/ 6 /$, ist auch die Erzeugung von KugelstoBwellen im LabormaBstab möglich. Weitgehend vergleichbar hiermit ist die Unterwasserfunkenentladung, verg $1 . / 7 /$, die schon fruhzeitig zur Materialverformung eingesetzt wurde. Bei der Fokussierung der KugelstoBwellen mit einem Ellipsoidreflektor können im zweiten Fokus leicht Drucke im Kilobarbereich erzielt werden. Diese Methode führte erstmalig zur erfolgreichen extrakorporalen /8/ StoBwellenlithotripsie.

Als mechanische Methoden zur StoBwellenerzeugung in Flüssigkeiten, vergl. 19/, werden u.a. GasMembranstoBrohre eingesetzt, um zunächst eine Gas-StoBwelle zu erzeugen, deren Druck sodann über Reflexion auf eine Flüssigkeitssäule übertragen wird. Ebenso können MembranstoBrohre auch direkt zur Erzeugung von StoBwellen in Flüssigkeiten $/ 10 /$ verwendet werden. SchlieBlich ist auch der direkte Kolbenschlag, vergl. $19 /$, oder der Wasserschlag (analog dem hydraulischen Widder) zur StoBwellenerzeugung geeignet. Allerdings besitzen sämtliche der bisher genannten Methoden den Nachteil einer vergleichsweise geringen Reproduzierbarkeit.

Dies ist nicht der Fall bei der piezoelektrischen und der elektromagnetischen StoBwellenerzeugung.

Im piezoelektrischen Fall wird an eine Piezokeramikplatte oder eine entsprechende kalottenförmige Anordnung ein elektrischer Spannungssprung angelegt. Hieraus resultiert ein momentaner mechanischer Spannungszustand $/ 11 /$, der zu Entlastungswellen führt, die sich von der Generatoroberfläche in die Piezokermik ausbreiten und gleichzeitig mit der Abstrahlung einer Druckwelle in die angrenzende Flüssigkeit verbunden sind. Durch mechanische Anpassung auf der flüssigkeitsabgewandten Seite ist die Abstrahlung eines reinen Uberdruckpulses im erzeugenden Querschnitt möglich. Im Fokus entsteht somit ein positiver Uberdruckpuls mit nachfolgendem Unterdrucksignal. Bei genügend hoher Druckamplitude erfolgt eine Aufsteilung zur StoBwelle auf dem Weg zum Fokus. StoBwellengeneratoren für medizinische Anwendungen sind aus mehreren 1000 Piezokeramikelementen zusammengesetzt und besitzen Durchmesser von $60 \mathrm{~cm}$, um im Fokus Drucke bis zu $1 \mathrm{kbar}$ bei Pulsdauern von etwa 0,5 us erzeugen zu können. Durch eine geeignete elektrische Ansteuerung ist es möglich, den Unterdruckanteil im Fokus zu reduzieren, vergl. $/ 1 /, 121$.

Bei der elektromagnetischen Erzeugung von StoBwellen in fiussigkeiten werden die starken, abstoBenden, magnetischen Kräfte zwischen zwei von entgegegengesetzt gerichteten Strömen durchflossenen Leitern ausgenutzt. Die entsprechende Anordnung $112 /$ besteht aus einer spiralförmig gewickelten Flachspule, vor der sich in geringem Abstand eine dünne Kupfermembrane befindet. Wird ein Hochspannungskondensator mittels Schailfunkenstrecke über die Flachspule entladen, so induziert der entstehende starke Stromsto $B$ einen entgegengesetzt gerichteten Kreisstrom (Wirbelstrom) in der Kupfermembrane. Diese erfährt hierdurch kurzzeitig eine starke AbstoBung, so daB in die angrenzende Flüs- sigkeit eine Druckwelle abgestrahlt wird. Aufgrund der Nichtlinearität der Wellenausbreitung steilt sich deren Wellenfront bis zur Ausbildung einer StoBfront mit einer Anstiegszeit von etwa $1 \mathrm{~ns} / 12 /, / 13 /$ bei 50 bar StoBdruck in Wasser auf. Mit einer entsprechenden Anordnung zur Erzeugung von ebenen StoBwellen im StoBrohr von $50 \mathrm{~mm} \emptyset$ können je nach Spulen- und Kondensatordaten StoBdrucke bis zu einigen 100 bar und Dauern von $0,5-10$ us ohne Fokussierung erzeugt werden. Aufgrund der sehr guten Reproduzierbarkeit wurden Messungen der stationären Stoßfrondicke $/ 13 /$ als Funktion des StoBdruckes durchgeführt, wobei sich in einem weiten Druckbereich Ubereinstimmung mit den unter Berücksichtigung der UItraschallabsorption und der Nichtlinearitätskonstanten $B / A$, vergl. $/ 13 /, / 14 /$, berechneten StoBfrontdicke ergab.

Druckverlauf und Amplitude können bei einem elektromagnetischen StoBwellengenerator einfach aus dem gemessenen elektrischen Stromsignal $/ 12 /$ berechnet werden. Wegen der groBen Druckreserven wird durch Fokussierung mit einer akustischen Linse auch die elektromagnetisch erzeugte StoBwelle erfolgreich für die Zertrümmerung von Konkrementen $/ 1 /, / 2 /$ eingesetzt, wobei der Generatordurchmesser von $\mathrm{ca} .12 \mathrm{~cm}$ nur so gro $B$ sein muB, wie es durch den kleinstmöglichen Abstand zum Konkrement von etwa $12 \mathrm{~cm}, \mathrm{z}$.B. einem Nierenstein, und dem offnungswinkel von mindestens $60^{\circ}$ erforderlich ist. Eine genaue Analyse /12/. zeigt, daß der akusto-elektrische Wirkungsgrad des elektromagnetischen Generators reziprok zur Generatorfläche zunimmt, d.h. eine möglichst kleine Generatorfläche von Vorteil ist.

Statt mit einer Linsenfokussierung kann der elektromagnetische Generator auch in Kalottenform $115 /$, d.h. selbstfokussierend aufgebaut werden. In diesem Fall erfolgt die Wellenaufsteilung auf dem Weg zum Fokus. Uberraschenderweise beobachteten wir bei Modellexperimenten /16/ mit Phantomsteinen eine nennenswerte Zertrümerungswirkung auch ohne voll ausgebildete StoBfront im Fokus. Dies kann sowohl auf Kavitationseinwirkung durch. stets vorhandenen Unterdruckanteil als auch auf die direkte mechanische Einwirkung der Druckwelle mit endlicher Steilheit zurückzuführen sein. Scherspannungen und "Zug"-wellen (durch Reflexion im Stein bei Wellenaustritt), die eine starke Steinbeanspruchung darstellen, sind an die Bedingung geknüpft, daB die Wellenanstiegszeit kurz ist gegenüber der Laufzeit im Stein. Eine voll ausgebildete StoBfront ist daher besonders wichtig, wenn Steinbruchstücke weiter zu zerkleinern sind. Bei früheren Druckmessungen mit piezokeramischen Sondenhydrophonen $/ 17 /$ ergab sich bei Druckpulsen von 100 bar eine sehr hohe Sondenlebensdauer, sobald jedoch die selben Druckpulse nach einem Laufweg von $15 \mathrm{~cm}$ durch Aufsteilung eine scharfe StoBfront entwickelt hatten, wurden die Hydrophone schon durch etwa drei Expositionen zerstört.

Hinsichtlich der Wirkung des Unterdrucks und der Kavitation im Fokus, müssen die Gesichtspunkte berücksichtigt werden, daß einerseits die Kavitation zur Steinzertrümmerung beiträgt $/ 3 /$, andererseits auch zu Gewebsverletzungen, vergl. $/ 18 /$, führen kann. Hier ermöglicht der elektromagnetische Generator durch geeignete Wahl der zeitlichen Form des Generatorstroms, vergl. /4/, und damit des primären Druckimpulses eine Einstellung der jeweils gewünschten Pulsformen im Fokus. 
Zur Reduktion der Unterdrucksignalanteile auf der Achse zum Fokus kann das bereits. grundsätzlich beschriebene Verfahren der Randabschattung durch entsprechende Spulenabgriffe am elektromagnetischen Generator /4/ realisiert werden.

Die beschriebenen Stoßwellengeneratoren unterscheiden sich hinsichtlich ihrer Fokusabmessungen. Dies ist durch unterschiedliche offnungswinkel und Pulsdauern bedingt. Kleine Fokusabmessungen von etwa $5 \mathrm{~mm} \emptyset$ werden bei großer offnung und kurzer Generatorpulsdauer (piezoelektrisches System) beobachtet 127 /, während bei Unterwasserfunken- und elektromagnetischen Generatoren infolge geringer offnung und längerer Generatorpulsdauer Fokusdurchmesser von bis zu $20 \mathrm{~mm}$ auftreten. Wegen der geringen Gesamtenergie der akustischen Pulse des piezoelektrischen Generators ist die stärkere Fokussierung bei der Steinzertrümerung unbedingt erforderlich. Andererseits sind bei geringerer $\mathrm{Ge}-$ samtenergie und großer Offnung kavitationsbedingte Schmerzwirkungen, insbesondere an der Hautoberfläche im Eintrittsbereich vermeidbar, vergl. auch /28/.

\section{StoBwellenmeBmethoden}

Die Bestimmung von StoBwellendruck- oder Schnelleamplituden in Flüssigkeiten erfordert infolge der großen Pulsdauer und der gleichzeitig extrem kurzen Anstiegszeiten in der StoBfront eine groBe $\mathrm{Hy}-$ drophonbandbreite. AuBerdem sind die Hydrophone der zerstörenden Wirkung der StoBwelle selbst durch induzierte Scher- und Zugspannungen sowie ebenso der zerstörenden Wirkung der durch die nachfolgende Unterdruckwelle bedingten Kavitation ausgesetzt.

Diese Nachteile können z.B. durch direkte Erfassung der sich unter Druck ändernden optischen Brechungseigenschaften der Flüssigkeiten vermieden werden. Hier wird schon seit langer Zeit die schlierenoptische Abbildung eingesetzt. Allerdings ist eine quantitative schlierenoptische Druckbestimmung auBerordentlich schwierig. Demgegenüber ermöglicht eine neue optische Glasfaser-Hydrophonanordnung /19/ die unkomplizierte und sichere Bestimmung von kurzzeitigen Dichteänderungen in Flüssigkeiten und damit von kurzzeitigen Drucksignalen.

Gegenüber dieser neuen Methode der Druckmessung ist der Einsatz piezoelektrischer Hydrophone trotz begrenzter Lebensdauer bei hohen StoBdrucken weit verbreitet.

\section{"Flächenhafte" piezoelektrische Hydrophone}

Höchste Zeitauflösung verlangt geringste Detektordimensionen. Sofern die StoBwelle eben ist, bzw. einen genügend großen Krümmungsradius besitzt, kann die Detektion "flächenhaft" erfolgen. Dies soll in einfacher Weise für den Fall eines dicken Piezokristalls diskutiert werden. vergl. /12/, 113/. Bei senkrechtem Auftreffen eines Drucksignals oder eines StoBwellen-Drucksprunges auf den ebenen Piezokristall, wird bei der Reflexion eine Longitudinalwelle mit steiler Front in den Kristail abgestrahlt.

Infolge des Piezoeffekts sind mit den Fronten des Drucksprunges Polarisationsladungen verknüpft, die sich mit Schallgeschwindigkeit durch den Kristall bewegen. Bei geeigneter kapazitiver Auskopplung und niederohmigem AbschluB influenzieren die bewegten Polarisationsladungen Stromsignale deren Zeitverlauf ein getreues Abbild des akustischen Drucksprunges ist. Da für die Zeitauflösung allein die Kristall-Vorder- oder Rückseite verantwortlich ist, bezeichnet man diese Methode auch als Oberflächenabtastung bzw. Vorderseiten- oder Rückseitenabtastung. Die obere Frequenzgrenze ist hierbei durch die Parallelität der Kristalloberfläche gegenüber der Wellenfront bzw. der Größe des Krümmungsradius der Wellenfront gegenüber dem effektiven Durchmesser der Vorder- oder Rückseitenabtastung gegeben. Mit dieser Methode wurden die Anstiegszeiten in der StoBfront /13/ mit hoher Genauigkeit, d.h. einer Zeitauflösung besser als 0,1 ns bestimmt. Während diese piezoelektrische Methode zu hohen Frequenzen hin prinzipiell nur durch den Atomabstand im Kristall begrenzt ist, d.h. $\max$. ca. $10^{13} \mathrm{~Hz}$, ergibt sich eine Auflösungsgrenze bei tiefen Frequenzen durch die einfache Laufzeit im Kristall. Bei $5 \mathrm{~mm} \mathrm{Kri}$ stalldicke resultiert hieraus für eine Quarzscheibe eine untere Frequenzgrenze entsprechend der maximalen Pulsdauer von 1 Hs. D.h. eine möglichst hohe Bandbreite auch zu tiefen Frequenzen hin verlangt bei dieser Methode, die das Einschwingverhalten piezoelektrischer Platten ausnutzt, möglichst dicke Kristalle.

Im Gegensatz zum Prinzip der piezoelektrischen Vorderseiten- oder Rückseitenabtastung durch Strommessung, wird bei den bekannteren piezoelektrischen Hydrophonen das durch Kompression entstehende Spannungssignal gemessen. Aus diesem Grund darf ein Hydrophon groBer Bandbreite nur geringe Ausdehnungen besitzen. Bei ebenen Wellen und guter Paralleljustierung zwischen Hydrophon und Welle, kann eine piezoelektrische Schicht in Form einer dïnnen Kristallplatte, vergl. /20/, oder einer piezoelektrischen Polymerfolie (PVDF) direkt auf den Innenleiter einer Koaxialleitung geklebt werden, wobei die Gegenelektrode z.B. durch eine Leitsilberschicht oder als Metallbedampfung gebildet wird. Diese Hydrophone besitzen hohe Empfindlichkeit, zeigen jedoch wegen des endlichen Druckmessers des empfindlichen Elements und der Koaxialleitung eine scharfe Richtcharakteristik sowie Störungen der Pulsantwort (Schwankungen des Frequenzgangs) durch Randbeugung und Reflexionen. Diese Störungen werden beí dem Membranhydrophon /21/ vermieden. Anstelle eines flachen Piezoelements mit kleinem Durchmesser am Ende einer elektrischen Zuleitung ist bei diesem Hydrophon eine kreisförmige PVDFFolie von wenigen $u$ m Dicke in einen ringförmigen Rahmen von etwa $10 \mathrm{~cm}$ Durchmesser eingespannt. Die Folie wird lediglich im Zentrum in einem Bereich von etwa $0,5 \mathrm{~mm}$ Durchmesser polarisiert. Zuleitungen und Abschirmung werden als Metallaufdampfschichten ausgeführt. Wegen der verhältnismäBig guten akustischen Anpassung von PVDF an Wasser und wegen der sehr geringen Beugungs- und Reflexionsfehler durch Zuleitungen und Einspannung besitzen Membranhydrophone eine groBe Bandbreite von 0 - $30 \mathrm{MHz}$ (minimal Anstiegszeit ca. $10 \mathrm{~ns}$ ). Die Empfindlichkeit liegt im Bereich von $3 \mathrm{mV} / \mathrm{bar}$. Unter StoBwellenbedingungen werden nach längerer Zeit die metallischen Zuleitungsbahnen durch Kavitation zerstört. Ein Nachteil dieses Hydrophons ist seine beträchtliche Größe.

Neben piezoelektrischen flächenhaften Hydrophonen können StoBwellenmessungen auch mit Hilfe des elektrodynamischen Prinzips /11/ (Bändchenmikrophon) oder auch des elektrostatischen Prinzips bei Reflexion der Stoßwelle an einer Grenzfläche erfolgen. Insbesondere die elektrostratische Messung an der freien, die Stoßwelle reflektierenden Grenzfläche 123/ besitzt den Vorteil großer Bandbreite, der 
einfachen Möglichkeit der absoluten Eichung unabhängig von anderen Druckstandards sowie der unbegrenzten Lebensdauer unter StoBwellenbedingungen. Ein solches Hydrophon besteht aus einer Elektrode, die sich in geringem Abstand unmittelbar über der mit einer duinnen metallisierten Membran abgedeckten Flüssigkeitsoberfläche befindet. Diese Elektrode wird über einen Vorwiderstand auf etwa 50 Volt Vorspannung gebracht und mit dem hochohmigen Eingang eines Breitbandverstärkers verbunden. Aufgrund der bei der StoBwellenreflexion auftretenden Abstandsänderung entsteht ein schnelleproportionales Sondensignal, wobei die obere Grenzfrequenz durch die RC-Zeitkonstante der Sonde bestimmt ist. Die Eichung /23/ erfolgt durch entsprechende mechanische Schwingungsanregung der Sonde mit optisch bestimmter Amplitude und bekannter Frequenz.

\section{"Punktförmige" Hydrophone}

Piezoelektrische Drucksonden wurden lange Zeit durch Aufeinanderkleben von entgegengesetzt orientierten Piezokristallen in mm-Dimensionen hergestellt, wobei der Innenleiter des MeBkabels mit einer Metallfolie zwischen den Kristallen und der AuBenleiter mit einer Metallbeschichtung auf der AuBenseite des Kristallpakets verbunden wurde. Die Bandbreite dieser Hydrophone betrug in der Regel höchstens einige hundert $\mathrm{KHz}$.

Die erste piezoelektrische Sonde mit einer Bandbreite von einigen $\mathrm{MHz} / 24 /$ bestand aus einer auf einer Platinkugel von ca. 0,3 mm Durchmesser aufgesinterten Barium-Titanat-Schicht von etwa $0,1 \mathrm{~mm}$ Dicke. Die Platinkugel befand sich am Ende eines Platindrahtes als Innenelektrode, der in eine Glaskapillare eingeschmolzen war. Als AuBenelektrode diente eine eingebrannte Silberschicht. Bei Anstiegszeiten von 50 ns betrug die Empfindlichkeit /11/ etwa $5 \mathrm{mV} /$ bar. Diese Hydrophone werden jedoch in der voll ausgebildeten StoBwelle /17/ schnell zerstört. Es ist zu vermuten, daB die gesinterte Barium-Titanat-Schicht den hohen mechanischen Spannungsgradienten in der StoBfront nicht standhält (vergl. Wirkung auf Konkremente).

Ein ähnliches neues Hydrophon mit dem piezoelektrischen Polymer PVDF ist das Nadelhydrophon /20/. Der empfindliche Bereich dieses Hydrophons ist auf die mit PVDF bedeckte verrundete Spitze einer "Nadel" begrenzt, die in den Innenleiter einer dünnen koaxialen Zuleitung übergeht. Die Begrenzung des empfindlichen Bereichs auf die Spitze wird durch die nachträgliche Polarisation des PVDF-Materials 2.B. in einer stark lokalisierten Koronaentladung erreicht. Als AuBenleiter wird auch hier meistens Leitsilber verwendet. Anstiegszeiten von 50 ns bei einer Empfindlichkeit von $1 \mathrm{mV} /$ bar können leicht erreicht werden. Diese $\mathrm{Hy}$ drophone sind aufgrund der elastischen Eigenschaften des PVDF-Materials gegenüber der StoBwellenwirkung sehr beständig, allerdings wird der Leitsilberüberzug durch Kavitation zerstört, kann aber erneuert werden. Für einen glatten Frequenzgang, bzw. eine einwandfreie Impulsantwort, müssen bei der Herstellung dieser Hydrophone selbst kleinste Gasblasen im Polymermaterial und in der Leitsilberbeschichtung unbedingt vermieden werden.

Bei einem völlig anderen Meßprinzip, der optischen Sonde $/ 19 /$, werden ein komplizierter mehrschichtiger Aufbau, zunehmende Schwierigkeiten bei der weiteren Miniaturisierung und Verlängerung der Zuleitungen, auf Kosten eines höheren geräteseitigen Aufwandes vermieden. Bei diesem Hydrophon wird die druckbedingte Dichte und damit Brechungsindexer- höhung als MeBgröße bestimmt. Dies gelingt mit hoher räumlicher und zeitlicher Auflösung durch die opto-elektronische Erfassung der Lichtreflexionsänderung am Ende einer in das Schallfeld-eintauchenden Glasfaser. Hierzu wird Laserlicht in die Glasfaser eingekoppelt und das reflektierte Licht mit einem schnellen Fotodetektor in das elektrische Hydrophonsignal umgesetzt. Derzeit liegt die durch Laserrauschen bedingte Grenze der Druckauflösung bei etwa 1 bar mit einer Anstiegszeit von $50 \mathrm{nsec}$. Das Glasfaserhydrophon wird durch die StoBwelle nicht zerstört, ebenso konnten bisher keine Kavitationsschäden beobachtet werden. Ein neues empfindliches Faserende kann jederzeit leicht durch Anritzen und Brechen hergestellt werden. Glasfaserlänge und Durchmesser sind ohne wesentliche Einwirkung auf die Empfindlichkeit.

Die Zeitauflösung ist bei seitlicher Schallinzidenz durch den Glasfaserdurchmesser begrenzt, der auf wenige $\mu \mathrm{m}$ reduziert werden kann, bei senkrechter Inzidenz durch die Lichtwellenlänge entsprechend einer Maximalfrequenz von $3 \mathrm{GHz}$. Bei genügend breitbandiger elektrooptischer Signalverarbeitung kann das gesamte Stoßwellendrucksignal incl. des Stoßfrontanstiegs wiedergegeben werden.

\section{Kalibrierung}

StoBwellenhydrophone können ähnlich kalibriert werden wie andere Ultraschallwandler. Hierzu geeignet ist das über eine Strahlungsdruckwaage oder über die Oberflächenauslenkung $/ 25 /$ geeichte Ultraschallfeld eines piezoelektrischen Strahlers.

Ebenso geeignet sind die bekannten Reziprozitätseichmethoden von Schallfeldern /26/, die auch bei der Eichung von PVDF-Nadelhydrophonen /20/ unter Verwendung von PVDF-Strahlern eingesetzt werden. Mit den genannten UItraschallverfahren wird die Frequenzabhängigkeit der Empfindlichkeit bestimmt. Etwaige Phasenfehler, die die Pulsantwort beeinflussen, sind jedoch nur schwer quantitativ zu erfassen, wenngleich sie sich durch Schwankungen des Amplitudenfrequenzgangs indirekt bemerkbar machen.

Ein für Stoßwellenanwendungen grundsätzlich geeigneteres Kalibrierverfahren ist daher die Bestimmung der Drucksprung- oder auch der Pulsantwort des Hydrophons. Dies ist besonders wichtig für die Kalibrierung im Bereich von einigen 100 bar entsprechend den Bedingungen bei medizinischen StoBwellenanwendungen.

Solche Kalibrierungen sind in /6/ mit Hilfe von StoBwellen in FlüssigkeitstoBrohren bei StoBwellenerzeugung durch Kolbenschlag und GasmembranstoBrohren bis zu Drucken von 600 bar durchgeführt worden. Bei der GasstoBrohrmethode kann der durch Einkopplung in das WasserstoBrohr entstehende Druck aus dem statischen Gasdruck berechnet werden; bei der Kolbenschlagmethode folgt der erzeugte Druck aus der Kolbengeschwindigkeit. Eine weitere Druckkontrolle ist wegen der großen StoBwellendauer durch kommerzielle, geeichte Druckaufnehmer (Kistler-Sonden) möglich.

Eine Alternative zur Kalibrierung von Hydrophonen bis zu etwa 200 bar besteht in der Erzeugung von Druckimpulsen und Stoßwellen bekannter Amplitude mit dem elektromagnetischen Stoßwellengenerator 112/. Hier ergibt sich der Druck in der abgestrahlten ebenen Welle (bis auf eine korrigierbare Abweichung im Spulenzentrum) aus dem Generatorstrom und der Windungszahl pro Radius. 
Im Bereich niedriger Drucke führt das Einschwingverhalten einer momentan aufgeladenen Piezoplatte zur Abstrahlung eines Rechteckwellenzuges /11/ leicht bestimmbarer Amplitude in eine angrenzende Flüssigkeit. Die gleichzeitig im Inneren der Platte mehrfach reflektierte mechanische Welle erzeugt über den reziproken Piezoeffekt einen dreieckförmigen Spannungsverlauf. Aus dessen Amplitude und weiteren einfach zu bestimmenden elektrischen und mechanischen Daten kann die Amplitude der abgestrahl ten Rechteckwelle im Sinne einer Selbstreziprozitätseichung berechnet werden.

Eine einfache Kalibrierung bei hohen StoBwellendrucken ist im Fokus eines Stoßwellengenerators mit Hilfe der bereits beschriebenen kapazitiven Schne1le-Sonde /23/ möglich. Nach der Bestimmung des absoluten Schallfelddruckes (Schnelle) mit der Sonde wird der Wasserspiegel über dem Generator erhöht und die zu eichende piezoelektrische Drucksonde an den gleichen Ort gebracht.

SchlieBlich ist auch das optische Sondenhydrophon leicht eichbar. Da der Zusammenhang zwischen Druck und Brechungsindex in Wasser bekannt ist, kann die stationäre Anderung der Lichtreflexion durch Vergleich des optischen Sondensignals bei Wasser und z.B. einer Salzlösung mit leicht erhöhtem und ebenso bekanntem Brechungsindex bestimmt werden.

Insgesamt stehen somit eine Reihe von verschiedenen Kalibrierverfahren zum Vergleich zur Verfügung.

In diesem Bericht konnten nur die wichtigsten StoBwellenerzeugungs- und MeBverfahren diskutiert werden. Nähere Einzelheiten sind der aufgeführten Literatur zu entnehmen.

\section{Literatur:}

/1/ Urologische Steintherapie, ESWL und Endourologie

Herausg, von F. Eisenberger und K. Miller, Georg Thieme Verlag Stuttgart, New York 1987

12/ StoBwellenlithotripsie bei Harn- und Gallensteinen Herausg. von M. Ziegler, Springer Verlag 1987

13/ A Mechanism of Gallstone Destruction by Extracorporal Shock Waves

$M$. Delius and W. Brendel, Naturwissenschaften 75, 200-201, (1988)

14/ Formung fokussierter akustischer Druckpulse J. Staudenraus, K. Holdik, W. Eisenmenger Fortschritte der Akustik, DAGA'87, DPG, pp.445

15/ Underwater Explosions

R.H. Cole, Dover Publications, New York 1965

16/ Propagation and Focussing of Spherical Shock Waves produced by Underwater Microexplosions K. Takayama, H. Esashi, N. Sanada Proc. 14th Int. Symp. on Shock Tubes and Waves (R.D. Archer and B.E. Milton Eds), Sydney 1983

17/ Der Schallimpuls eines Flüssigkeitsfunkens E. Bailitis, Z. f. angew. Physik 9, 429 (1957)

18/ Berührungsfreie Harnsteinzertrümmerung F.Eisenberger, E. Schmiedt, Ch. Chaussy, K. Wanner, B. ForBmann, W. Hepp,

K. Pielsticker, W. Brendel Deutches Arzteblatt, Artzliche Mitteilungen 17, 1145, (1977)
Vergl, auch:

Eine Methode zur berühungsfreien Zertrümmerung von Nierensteinen durch StoBwellen

B. ForBmann, W. Hepp, Ch. Chaussy,

$\mathrm{F}$. Eisenberger, K. Wanner

Biomed. Techn. 22, 164 (1977)

19/ Stoßwellenfokussierung in Wasser

M. Mulller, Dissertation RWTH Aachen, 1987

Vergl. ebenso

Experimental Investigation on Focussing of

Weak Spherical Shock Waves in Water by Shallow Ellipsoidal Reflectors

M. Müller, Acustica 64, 85, (1987)

$110 /$ StoBwellen in Flüssigkeiten zur Untersuchung von Lösungsreaktionen, $A$. Jost Dissertation, Göttingen (1966)

111/ Eine Kontrollschallquelle für breitbandige Mikrofone zur Messung von Druckimpulsen in F)üssigkeiten

Acustica 12, 165, (1962)

112/ Elektromagnetische Erzeugung von ebenen OruckstöBen in Flüssigkeiten

W. Eisenmenger

Acustica, Akustische Beihefte, 12, 185, (1962)

113/ Experimentelle Bestimung der StoBfrontdicke aus dem akustischen Frequenzspektrum elektro. magnetisch erzeugter StoBwellen in Flüssigkeiten bei einem StoBdruckbereich von 10 ATM bis 100 ATM

W. Eisenmenger

Acustica 14, 187, (1964)

/14/ Nonlinear Acoustics

R.T. Beyer

Naval Sea Systems Command, 1974

115/ Einrichtung zur berührungsfreien Zertrümmerung von Konkrementen im Körper von Lebewesen P 3312014C2 (1983)

/16/ W. Eisenmenger, J. Staudenraus, unpublished, 1986

/17/ W. Eisenmenger, unpublished, 1961

/18/ Biological Effects of Shock-Waves: Kidney Damage by Shock Waves in Dogs, Dose Dependence M. Delius, G. Enders, J. Xuan, H.G. Liebich, W. Brende I

U1trasonics in Med.u.Biol. 14, 367, 1988

/19/ Optisches Sondenhydrophon J. Staudenraus, W. Eisenmenger Fortschritte der Akustik, DAGA 1988, DPG GmbH

120/ U1traschallwandler aus Polyvinylidenfluorid mit breitbandigem Ubertragungsverhalten und fokussierenden Eigenschaften

M. Platte

Acustica 54, 23, 1983

121/ The Characteristics of a PVDF Membrane Hydrophone

D.R. Bacon

IEEE Trans. Sonics. U1trason. Su-29, 18, (1982)

/22/ Siehe /11/

/23/ Elektrostatisches Sondenhydrophon mit einfacher Absoluteichung

W. Lawrenz, J. Staudenraus, W. Eisenmegner Fortschritte der Akustik, DAGA 1988, DPG GmbH 
124/ Miniature Piezoelectric Receivers

E.V. Romanenko,

Sov. Phys. Acoust. 3, 364, 1957

(Trans.. from J. Acoust. Soc. USSR)

125/ Ultraschall: FeldgröBen und ihre Bestimung

R. Reibold

Fortschritte der Akustik, DAGA'87, DPG, S79

/26/ Siehe /25/

127/ Comparative Measurement of the Physical Parameters of some Commercial Lithotriptors

J.E. Saunders

Vortrag: Conference for Ultrasonics in Biophy-

sics and Bioengineering

Allerton Park, Monticello, University of

Illinois at Urbana-Champaign, 1988

/28/ Physikalisch-medizinische Aspekte selbstfokussierter elektromagnetisch erzeugter StoBwellen

W. Eisenmenger

Urologe, Verhandlungsberichte, 39, 69, 1988 